Fairbrother, R. W., Parker, L. \& Eaton, B. R. (1954). J. gen. Microbiol. 10, 309-316

\title{
The Stability of Penicillinase-producing Strains of Staphylococcus aureus
}

\author{
By R. W. FAIRBROTHER, L. PARKER AND B. R. EATON \\ Department of Clinical Pathology, Manchester Royal Infirmary
}

SUMMARY : Penicillin-sensitive and penicillin-resistant colonies of Staphylococcus aureus, belonging to the same phage pattern, were found in six out of 200 apparently resistant cultures isolated from infective processes. The in vitro instability of penicillinase-producing strains of Staph. aureus was confirmed. It was found that growth with Streptococcus haemolyticus or in the presence of other antibiotics did not greatly influence the natural development of sensitive variants. This process, however, tended to be accelerated by growth at $44^{\circ}$, although some strains did not yield any sensitive variants in spite of prolonged incubation. Some inherited factor is considered to be primarily responsible for the production of penicillinase.

Two different types of resistance to penicillin are exhibited by Staphylococcus aureus. One type can be readily induced in vitro by serial subculture in increasing concentrations of the antibiotic; this change is reversible and is not associated with the formation of penicillinase. The other type has only been obtained under natural conditions; it tends to be permanent and is due to the production of penicillinase. These are quite distinct phenomena and require individual consideration.

Penicillinase-producing strains of Staph. aureus are being isolated from infected lesions with increasing frequency, especially in hospitals, and they present a serious clinical, as well as an important fundamental, problem. The origin of these strains has not been determined; both genetic and environmental factors have been incriminated, but conclusive evidence of their particular role has not been obtained. The only apparent difference between these resistant strains and sensitive strains is the production of penicillinase, which some workers consider to be a permanent characteristic of naturally occurring resistant strains. Recent work, however, indicates that these strains can give rise to sensitive variants.

Voureka (1948) obtained sensitive organisms by growing resistant strains of Staph. aureus in mixed culture with Streptococcus haemolyticus or other organisms; she suggested that this change was due to the activity of some substance produced by the associated organism. Workers at the Johns Hopkins University (Chandler, Davidson, Long \& Monnier, 1951; Monnier \& Schoenbach, 1951; Chandler \& Goltz, 1952) reported a marked diminution in penicillinase production, with the appearance of sensitive variants, by growing penicillin-resistant staphylococci in the presence of other antibiotics. Barber $(1949,1953)$ found that old cultures of penicillin-resistant staphylococci did not always contain a homogeneous population; by repeated subculture over a long period, sensitive variants could often be isolated from the resistant strains. She therefore considered that any variation in sensitivity, which 
occurred during the various procedures, was due to the natural selection of sensitive variants and not to any specific action on the penicillinase system. There is thus no doubt that penicillinase-producing strains of Staph. aureus can produce sensitive variants, but the mechanism responsible for this change is uncertain. As this is a problem of fundamental interest, it was decided to carry out further investigations, particularly on (1) the natural occurrence of staphylococcal variants in infective material, and (2) methods of increasing the natural instability of penicillinase-producing strains of Staph. aureus.

\section{METHODS}

All strains of Staph. aureus used were coagulase-positive by the tube test and those tested fermented mannitol and liquefied gelatin.

Sensitivity tests were carried out by: (1) the dried disk and strip technique (Fairbrother \& Martyn, 1951); (2) the serial dilution method, in which heavy inocula were used-1 drop ( $0.02 \mathrm{ml}$.) of a $24 \mathrm{hr}$. culture to $0.5 \mathrm{ml}$. of medium (c. 20 million organisms). Results in both methods were read after $18 \mathrm{hr}$. incubation.

Penicillinase tests. Agar plates were seeded uniformly with the Oxford strain of staphylococcus; sterile cylinders were placed on the medium and into them, first, 4 drops of an overnight broth culture of the test organism, and then 1 drop of penicillin solution (10 units/ml.) were pipetted; readings were made after overnight incubation. When this test failed to demonstrate the presence of penicillinase the organism was re-examined by a modification of the technique of Harper (1943). A suspension (0.5 ml.) in distilled water of the acetone and ether-treated cells was added to falling dilutions of penicillin in $0.5 \mathrm{ml}$. broth; the end-point was compared with a similar series of dilutions of penicillin to which distilled water only had been added. The presence of penicillinase is indicated by a difference in end-points.

Phage typing of the strains used was done at the Staphylococcal Reference Laboratory, Colindale.

\section{RESULTS}

\section{The presence of resistant and sensitive strains of Staphyloccocus aureus in the same lesion}

It is generally accepted that penicillinase-producing organisms cannot be produced in vitro from sensitive strains of Staph. aureus. It has, however, been suggested that penicillinase-producing mutants may arise from sensitive strains in vivo during treatment with penicillin; conclusive evidence to support this claim has not yet been produced. It is therefore surprising that few investigations on the occurrence of staphylococcal variants in vivo have been carried out. Barber \& Whitehead (1949) examined individual colonies of cultures made from infected material and isolated sensitive and resistant strains of Staph. aureus from the same patients on eleven occasions. The strains were phage-typed but, as one or both of the pair was untypable in eight cases, the results were inconclusive; in the majority of cases the organisms appeared to belong to different types. 
In routine work, sensitivity tests by the disk or similar technique are done either on the original material or, more frequently, on pure cultures prepared indiscriminately from the original plate. Under such conditions the detection of variants is difficult, owing to the production of penicillinase by resistant organisms and consequent inactivation of the test penicillin. As a general rule the presence of penicillin-sensitive variants in a resistant culture of Staph. aureus can only be detected by the examination of the individual colonies. Accordingly, discrete colonies (15-50) were selected at random from 200 original cultures of infected material, found to be resistant in the routine test, and examined by the strip technique.

Occasional sensitive variants were found on thirteen occasions. In two cases, only one sensitive colony was found out of totals of forty and fifty respectively. It is possible that the number of cultures with sensitive variants would have been greater if more colonies had been selected from the original plates, as fifty colonies represent only an extremely small sample of the whole culture. The variants were checked for coagulase-production, and the sensitivity of the sensitive and resistant organisms determined by the serial dilution method. The resistant strains were only inhibited by at least 100 units penicillin/ml. and produced penicillinase, whereas the sensitive strains were inhibited by $\frac{1}{8}$ unit or less penicillin $/ \mathrm{ml}$. and did not form penicillinase.

The resistant and sensitive strains, as well as the original culture, were phage-typed. In five of the thirteen mixed cultures of penicillin-sensitive and penicillin-resistant staphylococci the two strains were of different phagepatterns and, in two, one of each pair was untypable. These findings strongly suggest that, in these seven cases, the two strains originated from different sources.

In six of the thirteen mixed cultures, however, the two strains had the same phage-pattern and the organisms of each pair probably originated from the same parent strain. Only occasional sensitive colonies were isolated in each instance from the infected material, which was not, however, examined in the initial stages of the infection. There was, therefore, no conclusive evidence to indicate the sensitivity to penicillin of the original infecting strain, or the mechanism by which the variants developed in vivo.

\section{In vitro development of sensitive variants from penicillinase-producing Staphylococcus aureus}

The natural instability of some penicillinase-producing strains of Staph. aureus was originally demonstrated by Barber (1949), who considered that, apart from repeated subculture, there was no way of accelerating this natural tendency. Growth with other organisms or in the presence of other antibiotics made no significant difference. In view of other claims, the in vitro development of sensitive variants from resistant organisms was investigated by three different methods: growth with Strep. haemolyticus; growth in the presence of other antibiotics; growth at $\mathbf{4 4 ^ { \circ }}$.

Groroth in the presence of Streptococcus haemolyticus. Following the technique of Voureka (1948), penicillinase-producing strains of Staph. aureus 
were grown in mixed culture with a penicillin-sensitive strain of Strep. haemolyticus (group A). Twenty resistant strains of Staph. aureus were examined and seven different strains of Strep. haemolyticus used. After incubation at $37^{\circ}$ for $24 \mathrm{hr}$., subcultures of the original and the mixed staphylococcal cultures were prepared on blood agar plates. After overnight incubation, ten to fifty colonies were tested for penicillin sensitivity by the strip technique. Eighteen of the twenty strains of Staph. aureus yielded only resistant colonies. From one strain, however, a few sensitive colonies were obtained, but these were isolated in similar proportions from both the original and treated cultures. The sensitive variant belonged to the same phage-type as the resistant organism. With another strain, one sensitive colony was isolated from the original culture but none was isolated from the treated culture, fifty colonies being examined in each case. These findings support the view of Barber that the development of sensitive variants from penicillin-resistant strains of Staph. aureus after mixed culture is due to the natural instability of these strains and not to the specific action of any substance produced by the other organisms.

Growth in the presence of other antibiotics. Using the technique of Chandler et al. (1951) strains of Staph. aureus, which were highly resistant to penicillin but sensitive to the other antibiotics, were grown in broth containing increasing concentrations of the other antibiotics. Sensitivity tests by the serial dilution method were done before and after the treatment (Table 1). In this series,

\section{Table 1. The effect of serial subcultivation in increasing concentrations of other antibiotics on penicillin resistance of Staphylococcus aureus}

\begin{tabular}{|c|c|c|c|c|}
\hline \multirow[b]{2}{*}{ Antibiotic* } & \multirow[b]{2}{*}{$\begin{array}{l}\text { No. of } \\
\text { strains } \\
\text { tested }\end{array}$} & \multirow[b]{2}{*}{$\begin{array}{c}\text { No. of } \\
\text { subcultures }\end{array}$} & \multicolumn{2}{|c|}{ Change in resistance } \\
\hline & & & $\begin{array}{l}\text { Increase to } \\
\text { homologous } \\
\text { antibiotic }\end{array}$ & $\begin{array}{l}\text { Decrease to } \\
\text { penicillin }\end{array}$ \\
\hline Aureomycin & 6 & 10 to 20 & 4- to 8 -fold & No change \\
\hline Terramycin & 3 & 10 to 15 & 8- to 16 -fold & No change \\
\hline Chloramphenicol & 1 & 10 & 4-fold & No change \\
\hline Streptomycin & 11 & 9 to 12 & $64-$ to 32,000 -fold & $\begin{array}{c}\begin{array}{c}7=\text { no change } \\
3=4-\text { to } 16-\text { fold } \\
1=512 \text {-fold }\end{array} \\
1=5\end{array}$ \\
\hline
\end{tabular}

* Aureomycin-initial concentration $=0.125 \mu \mathrm{g} . / \mathrm{ml}$. increasing to a maximum of $8 \mu \mathrm{g} . / \mathrm{ml}$. Terramycin-initial concentration $=0.5 \mu \mathrm{g} . / \mathrm{ml}$. increasing to a maximum of $8 \mu \mathrm{g} . / \mathrm{ml}$. Chloramphenicol-initial concentration $=8 \mu \mathrm{g} . / \mathrm{ml}$. increasing to $32 \mu \mathrm{g} . / \mathrm{ml}$. Streptomycininitial concentration $=1 \mu \mathrm{g} . / \mathrm{ml}$. increasing to a maximum of $32,000 \mu \mathrm{g} . / \mathrm{ml}$.

alteration in resistance to penicillin was observed only after serial subculture in broth containing increasing concentrations of streptomycin (initial concentration $1 \mu \mathrm{g} . / \mathrm{ml}$. and increasing 64 - to $32,000-$ fold), when four of eleven strains showed an increase in sensitivity to penicillin. This increased sensitivity was only slight in the case of three strains, from two of which a few sensitive colonies (one among twenty) were detected on colonial examination of cultures prepared both before and after streptomycin treatment. Subculture in the streptomycin broths had not affected their frequency. One resistant strain (Law), however, exhibited a marked transformation; after ten subcultures in 
the presence of streptomycin (final concentration $8192 \mu \mathrm{g} . / \mathrm{ml}$.), it became completely sensitive to penicillin. The original culture had proved to be unstable, as it yielded a few sensitive colonies, but a resistant colony had been selected for the test. After serial subculture in the presence of streptomycin, the resistance to which increased $\mathbf{3 2 , 0 0 0 - f o l d , ~ t w e n t y ~ o u t ~ o f ~ t w e n t y ~ c o l o n i e s ~}$ were highly sensitive to penicillin. With a mass culture, the disk technique gave a wide clear zone and there was no production of penicillinase. The resistant and sensitive organisms had the same phage-type.

In view of these results, two more resistant colonies were selected from the original 'Law' culture and subjected to similar treatment. In both cases, the result was quite different. There was a 16,000 -fold increase in resistance to streptomycin, but the sensitivity to penicillin was unaltered. Chandler \& Goltz (1952) reported a similar inconsistency in the behaviour of a resistant strain of Staph. aureus. They found that one strain (strain 8) remained unchanged after transfer through aureomycin broth, and yet, in an earlier investigation, Chandler et al. (1951) had found that this strain permanently lost its capacity to produce penicillinase after a similar course of treatment.

These results indicate that the action of other antibiotics on penicillinaseproducing strains of Staph. aureus is erratic and unpredictable. It seems more probable that the development of sensitive variants is due to the inherent instability of these strains rather than to any specific action of the other antibiotics on the penicillinase mechanism.

Growth at $44^{\circ}$. Since enzyme activity diminishes on increasing the temperature above the range of optimal activity, it was considered that, by incubation above $37^{\circ}$, the conversion of penicillinase-producing strains of Staph. aureus into sensitive variants might be accelerated. In consequence, serial subcultures in broth of resistant strains were incubated in a water-bath at $44^{\circ}$. This was found to be the highest temperature that would regularly permit active growth of these strains.

Resistant strains, freshly isolated from active infections, were selected for the trial. Subcultures of a resistant colony were prepared on blood agar or agar plates. If twenty out of twenty colonies were found to be resistant, a known resistant colony was replated and, if all tested colonies of the subculture proved to be resistant, one of these was subcultured into $10 \mathrm{ml}$. broth in a 1 oz. screw-capped bottle and incubated at $44^{\circ}$. At intervals usually of 1-3 days serial subcultures were made in broth by means of a large loop; at the same time plate cultures were generally incubated at $37^{\circ}$ for sensitivity tests. Growth occurred readily in the early subcultures but tended to become slower and less marked in the later subcultures. Six different strains were investigated by this technique. In one case, a single-cell culture was also examined; in another, two separate colonies were tested and, with a third strain, a series of subcultures was also incubated at $37^{\circ}$ under otherwise identical conditions. The results are given in Table 2.

With the ' $L$ ' strain sensitive variants were first isolated after thirteen transfers during 24 days, when seven out of sixty colonies proved to be sensitive. On further incubation, the number of sensitive colonies increased 
variants of this strain were more numerous and appeared more rapidly in the series incubated at $\mathbf{4} 4^{\circ}$.

In all cases, whenever sensitive variants were isolated, the resistant and sensitive organisms were tested for coagulase and penicillinase, fermentation of mannitol, liquefaction of gelatin and phage-type. Invariably there was complete agreement except in the case of penicillinase-production, when the sensitive variants always gave negative results. Periodic examinations were also made on organisms which remained persistently resistant; these invariably behaved in the same manner as the parent strain. The sensitive variants tended to show definite colonial changes, becoming less pigmented and flatter, but these changes were not always associated with a change in sensitivity to penicillin.

\section{DISCUSSION}

These results confirm the view that cultures, and indeed individual colonies, of penicillinase-producing strains of Staph. aureus do not always contain a homogeneous population. Some strains exhibit a natural tendency to produce sensitive variants, the incidence of which is accelerated by repeated subcultivation at $44^{\circ}$ but is not greatly affected by growth in the presence of a haemolytic streptococcus or another antibiotic.

These findings cannot readily be explained by the concept of physiological adaptation to the modified environment. Some form of mutational change seems a more probable explanation. It is therefore suggested that some inherited factor controls the production of penicillinase by naturally occurring resistant strains but, in view of the limited knowledge of bacterial genetics and the primitive character of the staphylococcus, it is doubtful if this factor should be termed a gene. Staphylococci propagate by fission, but it is probable that the bacterial cells do not always divide into two identical daughter cells. In consequence any factors originally present may be unevenly distributed among the progeny.

When resistant strains of Staph. aureus possess relatively large amounts of the penicillinase factor, the development of sensitive variants may not occur, even at $44^{\circ}$. In some cases, e.g. ' $W$ ' single-cell culture and 'LAW 2', a few sensitive variants may be produced erratically but these are unable to establish themselves, being overwhelmed by the resistant, penicillinaseproducing organisms. On the other hand, strains possessing only a small proportion of this factor may readily produce sensitive variants, particularly if the environmental conditions are suitable. Growth at $44^{\circ}$ probably accelerates the development of sensitive variants by retarding the rate of growth of the resistant organisms. The production of penicillinase is decreased but not inhibited by incubation at $44^{\circ}$. Penicillinase-producing strains of Staph. aureus are frequently unstable and, while the development of sensitive variants is probably dependent on inherited factors, this process can be accelerated by modifying the conditions of growth.

We wish to thank Dr R. E. O. Williams and Miss Joan E. Rippon for phage-typing the various strains of Staph. aureus, and Dr K. I. Johnstone for preparing the singlecell culture of the ' $W$ ' strain. 


\section{REFERENCES}

BArber, M. (1949). The incidence of penicillin-sensitive variant colonies in penicillinase-producing strains of Staphylococcus pyogenes. J. gen. Microbiol. 3, 274.

Barber, M. (1953). The effect of serial passage in other antibiotics on penicillinaseproducing staphylococci. J. gen. Microbiol. 8, 104.

BARBER, M. \& WhITEHEAD, J. E. M. (1949). Bacteriophage types in penicillin-resistant staphylococcal infection. Brit. med. J. ii, 565.

Chandler, C. A., Davidson, V. Z., Long, P. H. \& Monnier, J. J. (1951). Studies on resistance of staphylococci to penicillin: The production of penicillinase and its inhibition by the action of aureomycin. Johns Hopk. Hosp. Bull. 89, 81.

Chandler, C. A. \& Goltz, E. von Der (1952). Studies on the effect of aureomycin on the production of penicillinase by staphylococci. Johns Hopk. Hosp. Bull. 91, 475.

Fairbrother, R. W. \& Martyn, G. (1951). The dise technique for determining sensitivity to the antibiotics. J. clin. Path. 4, 374.

HarPer, G. J. (1943). Inhibition of penicillin in routine culture media. Lancet, ii, 569.

Monnier, J. J. \& Schoenbach, E. B. (1951). The resultant sensitivity of microorganisms to various antibiotics after induced resistance to each of these agents. Antibiotics and Chemotherapy, 1, 472.

Voureka, A. (1948). Sensitization of penicillin-resistant bacteria. Lancet, i, 62.

(Received 29 September 1953) 